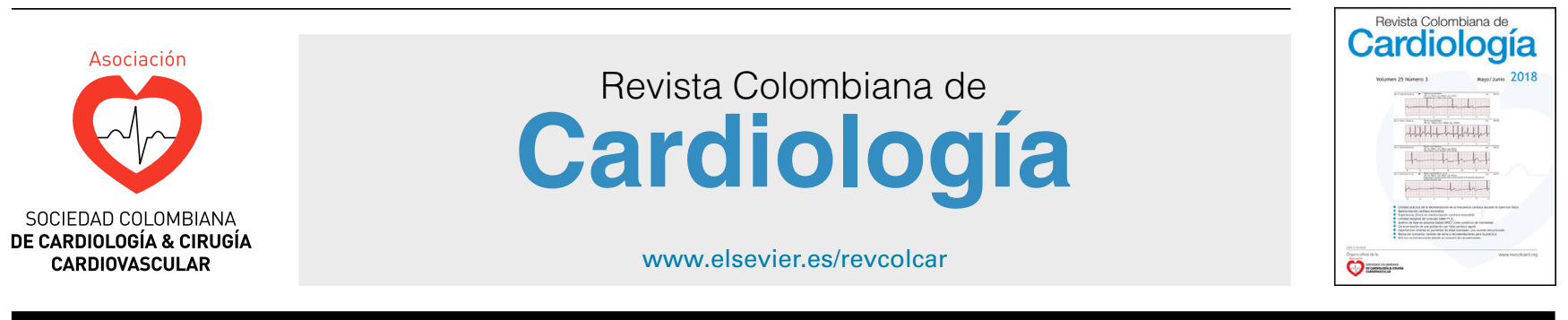

CARDIOLOGÍA DEL ADULTO - PRESENTACIÓN DE CASOS

\title{
Ablación septal con alcohol guiada con ecocardiograma para el manejo de la cardiopatía hipertrófica obstructiva
}

\author{
Celin Malkun Paz*, Pedro Graziano Sánchez y Manuel Rojano Ruiz
}

Clínica General del Norte, Barranquilla, Colombia

Recibido el 11 de septiembre de 2017; aceptado el 22 de marzo de 2018

Disponible en Internet el 21 de julio de 2018

\section{PALABRAS CLAVE \\ Miocardiopatía hipertrófica; Ablación septal; \\ Alcohol}

\section{KEYWORDS}

Hypertrophic cardiomyopathy; Septal ablation; Alcohol

\begin{abstract}
Resumen
Objetivo: Reportar el primer caso de ablación septal con alcohol guiada por ecocardiografía realizada en la Costa Atlántica.

Material y métodos: Paciente femenina de 74 años de edad con cuadro clínico de disnea, dolor anginoso y síncope recurrente, en quien se diagnosticó hipertrofia septal asimétrica con obstrucción dinámica con gradiente de $60 \mathrm{~mm} \mathrm{Hg} \mathrm{y} \mathrm{movimiento} \mathrm{anterior} \mathrm{sistólico} \mathrm{de} \mathrm{la} \mathrm{valva} \mathrm{anterior}$ de la mitral. Se le indicó ablación septal con alcohol.

Resultados: Se cateteriza selectivamente arteria primera septal donde a través de ecocardiograma se evidencia que es la rama a ablacionar y se pasan $1,5 \mathrm{ml}$ de alcohol, con lo cual se produce adecuada interrupción de flujo y caída del gradiente de presiones. Procedimiento exitoso sin complicaciones.

Conclusiones: Se reporta el primer caso de ablación septal con alcohol guiado mediante ecocardiograma, realizado en la costa Atlántica.

(c) 2018 Sociedad Colombiana de Cardiología y Cirugía Cardiovascular. Publicado por Elsevier España, S.L.U. Este es un artículo Open Access bajo la licencia CC BY-NC-ND (http:// creativecommons.org/licenses/by-nc-nd/4.0/).
\end{abstract}

Echocardiography-guided alcohol septal ablation for the management of hypertrophic obstructive cardiomyopathy

\section{Abstract}

Objective: To report the first case of echocardiography-guided alcohol septal ablation carried out in the Costa Atlantica, Colombia.

Material and methods: The case concerns a 74 year-old female patient with a clinical picture of dyspnoea, agina pain, and recurrent syncope. She was diagnosed with asymmetric septal hypertrophy with a dynamic obsrtuction with a gradient of $60 \mathrm{mmHg}$ and a systolic anterior motion of the anterior mitral valve. Septal ablation with alcohol was indicated.

\footnotetext{
* Autor para correspondencia.

Correo electrónico: cmalkun@yahoo.com (C. Malkun Paz).
} 
Results: The primary septal artery that was catheterised using echocardiography showed that it was the branch ablate, and $1.5 \mathrm{ml}$ alcohol was injected. This led to an adequate interuption of the flow and drop in the gradient pressures. The procedure was successful with no complications. Conclusions: The first case is reported of echocardiography-guided alcohol septal ablation carried out in the Costa Atlantica.

(c) 2018 Sociedad Colombiana de Cardiología y Cirugía Cardiovascular. Published by Elsevier España, S.L.U. This is an open access article under the CC BY-NC-ND license (http:// creativecommons.org/licenses/by-nc-nd/4.0/).

\section{Introducción}

La cardiopatía hipertrófica, descrita inicialmente por Teare ${ }^{1}$ en 1958, ha sido estudiada en forma exhaustiva, llegando a reconocerse como una patología cardiaca de origen genético, autosómico dominante, que afecta a una de cada 500 personas de la población general ${ }^{2,3}$. Presenta diferentes subtipos genéticos que pueden producir grados variables de afección al músculo cardiaco y en un $25 \%$ de estos casos puede causar una obstrucción significativa en el tracto de salida del ventrículo izquierdo ${ }^{4}$, cardiopatía hipertrófica obstructiva y cardiopatía hipertrófica no obstructiva, según se produce o no una obstrucción dinámica al tracto de salida del ventrículo izquierdo. La cardiopatía hipertrófica obstructiva se caracteriza por una hipertrofia asimétrica en la porción basal del septum interventricular, con reducción de la distancia entre la pared posterior del ventrículo izquierdo y el septum, acompañada de un movimiento anterior sistólico de la válvula mitral, que genera obstrucción dinámica en el tracto de salida del ventrículo izquierdo, en algunos casos con un gradiente significativo y sintomatología importante, predominantemente al esfuerzo, como disnea, angina o síncope, o incluso muerte súbita ${ }^{5}$.

La miomectomía quirúrgica, descrita en $1961^{6,7}$, ha sido el manejo estándar de la cardiopatía hipertrófica obstructiva en pacientes con obstrucción severa o muy sintomáticos. Las indicaciones para reducción de la hipertrofia septal incluyen presencia de obstrucción severa del tracto de salida del ventrículo izquierdo (gradiente de presión sistólica mayor o igual a $50 \mathrm{~mm} \mathrm{Hg}$ en reposo o inducida) y persistencia de síntomas (angina, disnea y/o síncope) a pesar de tratamiento médico óptimo ${ }^{7}$.

Desde el primer procedimiento realizado por Sigwart ${ }^{8}$ en 1994, la ablación septal con alcohol mostró ser una excelente opción terapéutica no quirúrgica para pacientes seleccionados portadores de cardiopatía hipertrófica obstructiva. Este método busca reducir el grosor del septum hipertrófico, mediante la introducción directa de alcohol a través de un catéter balón en la arteria septal que irriga el área hipertrófica, a fin de producir un infarto "controlado" y adelgazar dicha zona y, en consecuencia, reducir el gradiente del tracto de salida del ventrículo izquierdo ${ }^{5,8,9}$.

\section{Caso}

Paciente femenina de 74 años de edad, con antecedente de hipertensión arterial y diabetes mellitus tipo 2 con cuadro clínico de disnea de esfuerzo en clase funcional III/IV según la clasificación de la New York Heart Association, refractaria a tratamiento médico con betabloqueadores y calcioantagonistas, y sintomatología persistente de dolor anginoso, síncope recurrente, disnea, palpitaciones y mareos. Mediante ecocardiograma transtorácico se le diagnosticó hipertrofia septal asimétrica (grosor del septum de $26 \mathrm{~mm}$ ) con obstrucción dinámica en el tracto de salida gradiente pico de $60 \mathrm{~mm} \mathrm{Hg}$ y movimiento anterior sistólico de la valva anterior de la mitral. Se corroboró el diagnóstico de cardiopatía hipertrófica obstructiva mediante cateterismo cardiaco, en el que a su vez se observó lesión moderada del tercio medio de la arteria descendente anterior. Se midió el flujo de reserva fraccional (FFR) con test de hiperemia, mediante adenosina intracoronaria, con un resultado de 0,88 no significativo, determinándose que la lesión no ameritaba angioplastia.

El caso fue presentado en junta médico-quirúrgica en la cual se concluyó que dada la edad de la paciente, la presencia de comorbilidades (hipertensión, diabetes tipo II, nefropatía hipertensiva, enfermedad vascular en miembros inferiores), podría ser tratada mediante ablación septal con alcohol.

\section{Procedimiento}

En concordancia con protocolo establecido en la institución, la paciente fue llevada a sala de hemodinámica y bajo sedación anestésica intravenosa se instaló un electrodo de marcapaso transitorio a través de punción yugular interna derecha. Se comprobó adecuada estimulación y captura y se dejó marcapaso a demanda con frecuencia de $60 \mathrm{lpm}$ y salida de $6 \mathrm{mV}$. Se hizo ecocardiograma transtorácico con equipo Vivid $\mathrm{E} 9$ XDclear, al igual que cateterismo izquierdo previa preparación de regiones inguinales mediante asepsia y antisepsia en forma usual. Se colocaron campos en zona de punción femoral con anestesia local con xilocaína al $2 \%$ sin epinefrina; luego de la inserción bilateral de introductores en ambas arterias femorales mediante técnica de Judkins en arteria femoral derecha e izquierda, se pasó heparina 10.000 unidades intravenosa. Por vía femoral izquierda se introdujo catéter pig-tail $6 \mathrm{~F}$ dejándolo en ápex del ventrículo izquierdo conectado a transductor de presiones. Por vía arterial femoral derecha se introdujo catéter guía Judkins izquierdo $6 \mathrm{~F}$. Se realizó angiografía selectiva de la coronaria izquierda; se localizó la primera rama septal. Por el catéter guía, se introdujo guía metálica de 0,014 pulgadas y $300 \mathrm{~cm}$ de largo, posicionándola en arteria primera de la rama septal y a través de ella se pasó balón de angioplastia Apex-Push Over The Wire $1.5 \mathrm{~mm}$ por $8 \mathrm{~mm}$, colocándose en tercio proximal de la primera septal. Se procedió a llenar balón a presión de 6 ATM dejándolo inflado a fin de ocluir la rama septal. Se retiró guía y se inyectó medio 


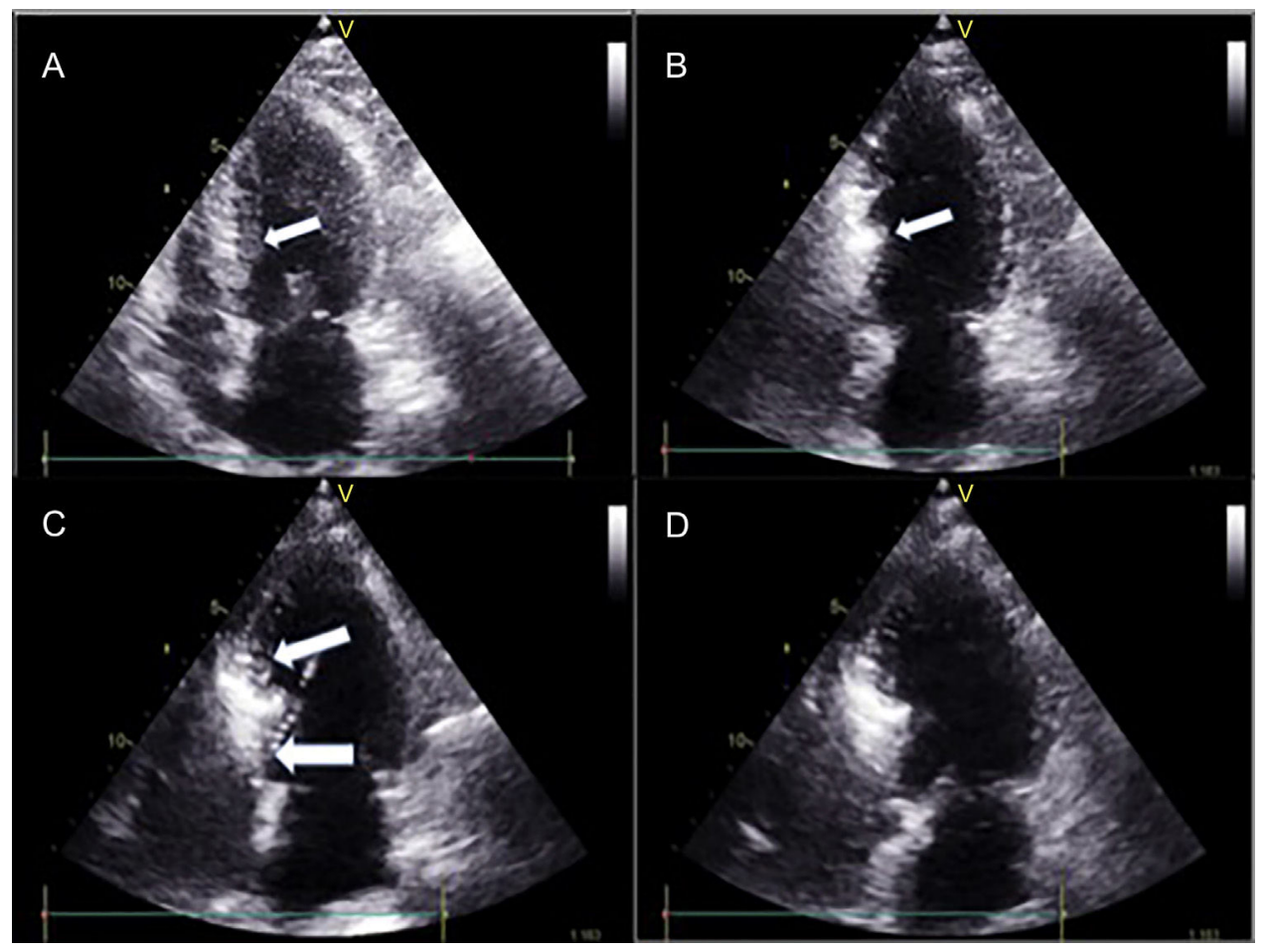

Figura 1 Ecocardiograma bidimensional que muestra la marcación del septum con medio de contraste hiperagitado. A) Imagen ecocardiográfica bidimensional que muestra el septum interventricular hipertrófico antes de pasar el medio de contraste. B) Inicio del paso del medio de contraste por la arteria primera septal comenzando a demarcar la zona septal de mayor irrigación. C) Inicio de la colocación del alcohol en la primera rama septal.

de contraste previamente hiperagitado, para realizar el contraste guiado por ecocardiograma transtorácico, delimitando la arteria septal donde se haría la ablación con alcohol (fig. 1). Con ayuda de este procedimiento se confirmó que la primera rama septal es la rama que da el flujo más significativo a la zona septal y es donde se debe hacer la ablación.

Mientras se mantenía inflado del balón a 6 ATM se pasaron $1,5 \mathrm{ml}$ de alcohol (etanol al 98\%) durante 5 minutos, después de los cuales se mantuvo inflado 5 minutos más y luego se lavaron los restos del alcohol en la arteria con solución salina. Se repitió angiografía por el catéter guía, observando oclusión completa de la primera rama septal (fig. 2).

Mediante toma de presiones interventriculares y ecocardiografía se confirmó la disminución significativa del gradiente en tracto de salida, evidenciando el éxito de la intervención. Se dio por terminado el procedimiento sin complicaciones. Posterior al procedimiento la paciente fue trasladada a la $\mathrm{UCl}$ para monitorización hemodinámica y electrocardiográfica durante 48 horas. Se realizó ecocardiograma de control 24 horas posteriores al procedimiento el cual mostró ausencia de gradiente infundibular (fig. 3).

\section{Discusión}

La miomectomía quirúrgica en casos de cardiopatía hipertrófica obstructiva es el método de elección (estándar dorado) para el manejo de esta patología; sin embargo, los centros quirúrgicos con amplia experiencia en el procedimiento son pocos, con resultados de morbimortalidad variables. En centros de referencia con alta experiencia la mortalidad es cercana al $2 \%$. Después de la miomectomía quirúrgica se tornan asintomáticos y el pronóstico a largo plazo de los supervivientes es excelente $\mathrm{e}^{10-12}$. En la ablación septal con alcohol, inicialmente se introduce un catéter en la primera y/o segunda rama septal y ahí se coloca el alcohol absoluto produciendo un infarto del miocardio controlado en el septum interventricular, con lo cual se reduce el engrosamiento sistólico del mismo y disminuye el gradiente en el tracto de salida.

Al igual que la miomectomía quirúrgica, la ablación septal con alcohol es eficaz en el alivio de la obstrucción, y su aplicación debería ser hecha por intervencionistas entrenados en el procedimiento ${ }^{11,12}$. El éxito de la ablación septal con alcohol depende de la selección del paciente, la experiencia del operador y la adecuada elección de la rama septal donde se colocará el alcohol. Son indicaciones para ablación septal con alcohol:

1) Pacientes con síntomas severos (angina de pecho clase III/IV de la Canadian Cardiac Society; disnea clase III o IV de la NYHA y/o síncope) refractarios a pesar de tratamiento medicamentoso óptimo.

2) Grosor septal mayor o igual a $15 \mathrm{~mm}$ y menor de 30 .

3) Obstrucción dinámica en el tracto de salida del ventrículo izquierdo gradiente $\geq$ a $30 \mathrm{~mm} \mathrm{Hg}$ en reposo o $\geq 50 \mathrm{mmHg}$ inducido.

4) Válvula mitral sin enfermedad intrínseca importante.

5) No requerimiento de otra cirugía cardiaca concomitante.

6) Arteria septal de calibre adecuado. 


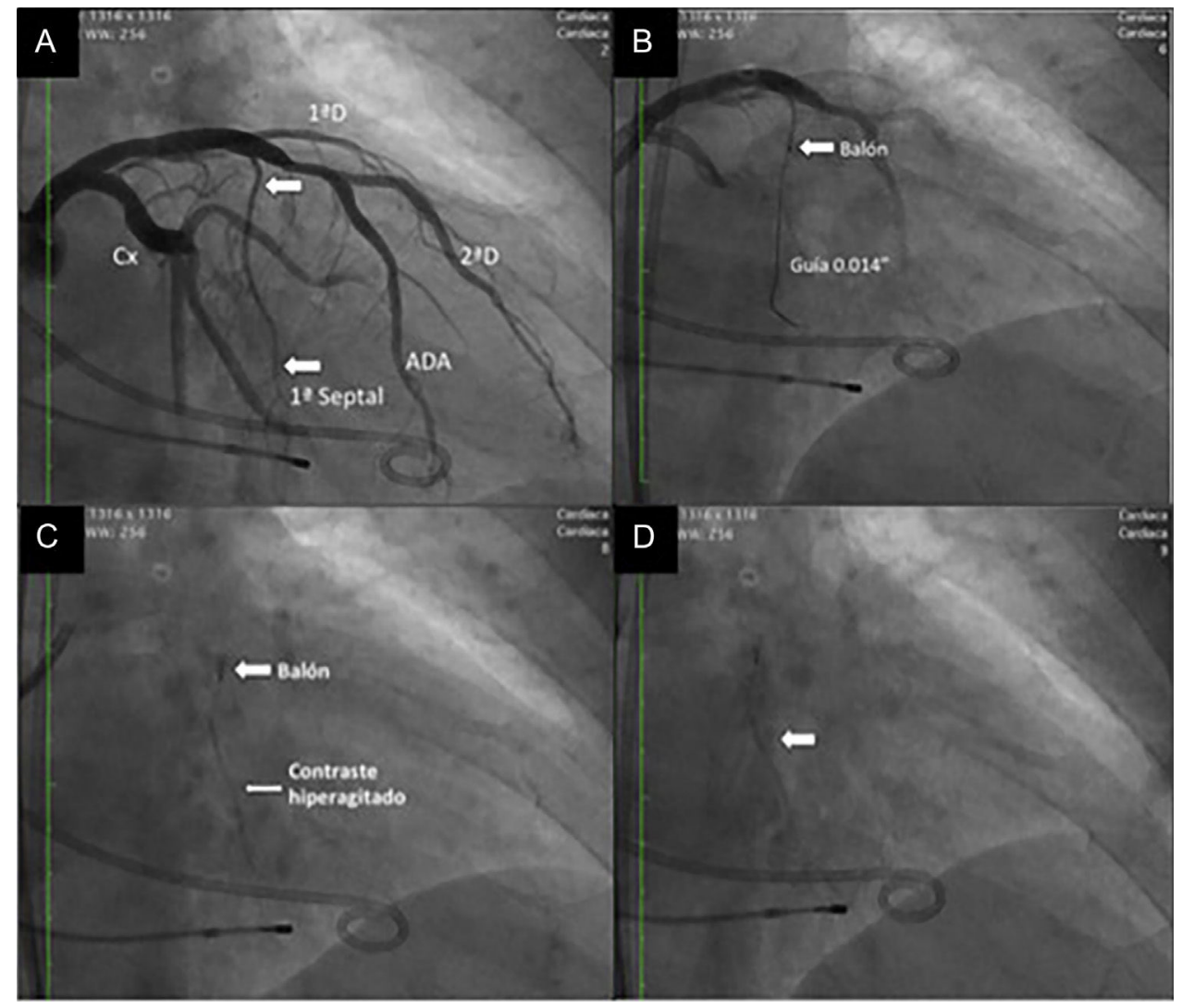

Figura 2 A) Angiografía de la arteria coronaria izquierda; las flechas demarcan la rama primera septal. B) Se posiciona la guía 0,014' en la rama primera septal y sobre esta se pasa el balón de angioplastia 1,5 x $8 \mathrm{~mm}$. C) Infusión del medio de contraste hiperagitado en la arteria primera septal. D) Infusión de alcohol en la rama primera septal.

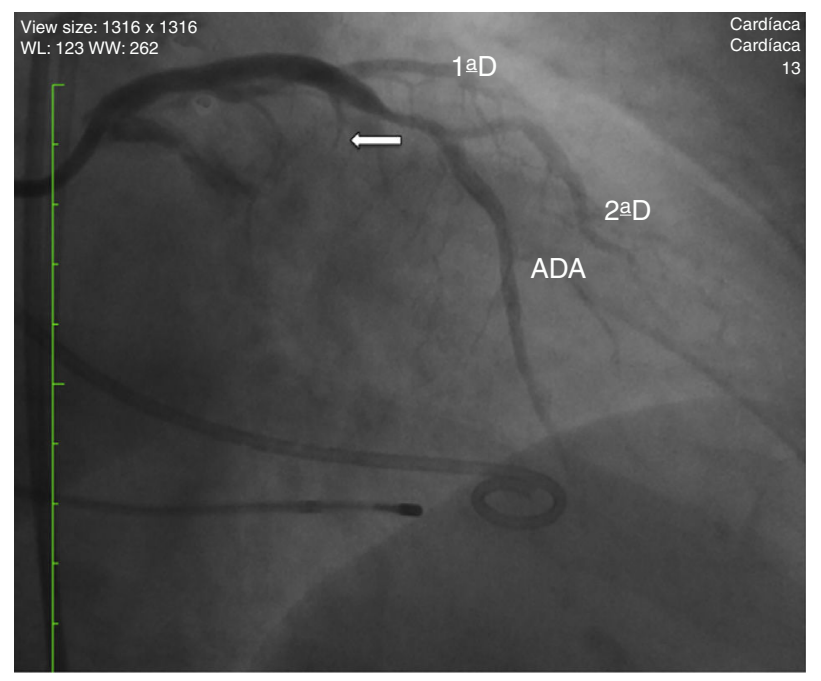

Figura 3 Resultado final; la flecha muestra oclusión total de la arteria primera septal posterior a la infusión del alcohol.

7) Aprobación del procedimiento por el paciente.

8) Pacientes que presenten contraindicación o intolerancia al uso de betabloqueadores o calcioantagonistas ${ }^{12}$.

La monitorización ecocardiográfica de este procedimiento fue introducida por Faber ${ }^{13}$ para intentar identificar con precisión la rama septal «diana» y poder evitar así la inyección del alcohol en una zona de miocardio no adecuada, como por ejemplo músculo papilar o pared libre del ventrículo izquierdo ${ }^{14}$.

Esta paciente presentaba además enfermedad coronaria moderada de la arteria descendente anterior y mediante estudio de flujo de reserva fraccional no requería intervención del mismo. Aunque no es una contraindicación absoluta, la enfermedad coronaria aterosclerótica, puede ser tratada mediante intervención coronaria previa a la ablación septal ${ }^{15}$, y en los casos que se presente enfermedad multivaso, donde está indicada la cirugía de revascularización coronaria, la miomectomía quirúrgica debería ser de elección.

Tanto la cirugía como la ablación septal percutánea deben ser consideradas como alternativas válidas en el manejo de la cardiopatía hipertrófica obstructiva. Las ventajas del tratamiento intervencionista percutáneo con ablación respecto a la ablación quirúrgica son las siguientes:

1) Se evita la circulación extracorpórea, con sus riesgos asociados (pacientes de edad avanzada con enfermedades extracardíacas concomitantes).

2) Tratamiento de obstrucción medioventricular u obstrucción combinada subaórtica y medioventricular.

3) Menor duración de la hospitalización.

4) Menor duración del período de recuperación. 


\section{5) Menor costo económico ${ }^{13}$.}

En los últimos años el número de procedimientos de ablación septal con alcohol se ha incrementado, siendo del orden de 5 a 10 veces mayor que el de la miomectomía ${ }^{13,14}$.

\section{Conclusiones}

Se presenta el caso de una ablación septal con alcohol guiada mediante ecocardiografía transesofágica para el manejo de cardiopatía hipertrófica obstructiva, la cual fue exitosa y permitió una disminución importante del gradiente dinámico y sin presencia de complicaciones. La guía apropiada por ecocardiograma durante el procedimiento, mejora los resultados y a su vez disminuye potenciales complicaciones. Se debe insistir en la elección adecuada de los pacientes.

\section{Conflicto de intereses}

Ninguno.

\section{Agradecimientos}

Al personal médico y paramédico del Departamento de Hemodinamia de la Clínica General del Norte de Barranquilla por el apoyo prestado para el reporte de este caso.

\section{Bibliografía}

1. Teare D. Asymmetrical hypertrophy of the heart in young adults. Br Heart J. 1958;20:1-8.

2. Maron BJ. Hypertrophic cardiomyopathy: a systematic review. JAMA. 2002;287:1308-20, http://dx.doi.org/10.1001/ jama.287.10.1308

3. Braunwald E, Lambrew CT, Rockoff D, et al. Idiopathic hypertrophic subaortic stenosis, I: a description of the disease based upon an analysis of 64 patients. Circulation. 1964;30 suppl IV:3-217.
4. Thierfelder L, Watkins H, MacRae C, Lamas R, McKenna W, Vosberg HP, et al. Alpha tropomyosin and cardiac troponin $\mathrm{T}$ mutation cause familial hypertrophic cardiomyopathy: A disease of the sarcomere. Cell. 1994;77:701-2.

5. Llamas-Esperon GA, Loera A, Sandoval S, Zamora A, Ramírez Robledo M, Varela S, et al. Ablación miocárdica septal transluminal percutánea en pacientes con miocardiopatía hipertrófica septal obstructiva, Resultados agudos y seguimiento a 3 años. Ach Cardiol Mex. 2009;79:33-40.

6. Morrow AG, Brockenbrough EC. Surgical treatment of idiopathic hypertrophic subaortic stenosis. Technic and hemodynamic results of subaortic ventriculomyotomy. Ann Surg. $1961 ; 154: 181-9$.

7. Kirklin JW, Ellis FH. Surgical relief of diffuse subvalvular aortic stenosis. Circulation. 1961;24:739-42.

8. Sigwart U. Non-surgical myocardial reduction for hypertrophic obstructive cardiomyopathy. Lancet. 1995;346:211-4.

9. Morrow AG, Reitz BA, Epstein SE, Henry WL, Conkle DM, Itscoitz SB, et al. Operative treatment in hypertrophic subaortic stenosis, Techniques, and de results of pre and postoperative assessments in 83 patients. Circulation. 1975;52:88-102.

10. Williams L, Rakowski H. Surgical myectomy for hypertrophic obstructive cardiomyopathy: the cut that heals. Circulation. 2013;128:193-7.

11. Braunwald E. Hypertrophic cardiomyopathy: the benefits of a multidisciplinary approach. N Engl J Med. 2002;347:1306-7.

12. Nagueh SF, Groves BM, Schwartz L, Smith KM, Wang A, Bach RG, et al. Alcohol septal ablation for the treatment of hypertrophic obstructive cardiomyopathy: a multicenter North American registry. J Am Coll Cardiol. 2011;58:2322-8.

13. Faber L, Seggewiss H, Gleichmann U. Percutaneous transluminal septal myocardial ablation in hypertrophic obstructive cardiomyopathy: results with respect to intraprocedural myocardial contrast echocardiography. Circulation. 1998;98:2415-21.

14. Seggewiss H, Rigopoulos A. Ablación septal en la miocardiopatía hipertrófica: situación actual. Rev Esp Cardiol. 2003;56: 1153-9.

15. Seggewiss H, Faber L, Meyners W, Bogunovic N, Odenthal HJ, Gleichmann U. Simultaneous percutaneous treatment in hypertrophic obstructive cardiomyopathy and coronary artery disease: a case report. Cathet Cardiovsac Diagn. 1998;44: 65-9. 